\title{
Using Ant Colony Optimization to Solve Train Timetabling Problem of Mass Rapid Transit
}

\author{
Jau-Ming Su ${ }^{1}$ Jen-Yu Huang ${ }^{2}$ \\ ${ }^{1}$ Department of Transportation Technology and Logistic Management, Chung-Hua University \\ ${ }^{2}$ Division of Traffic Engineering Institute of Civil Engineer, National Taiwan University
}

\begin{abstract}
The purpose of this research is to using ant colony optimization (ACO) to develop a heuristic algorithm to solve the train timetabling problem. This algorithm takes into consideration the trains scheduling in the transit period between peak period and off-peak period, the conflict resolving and the balance of in and out trains for each depot. A case study using the Taipei MRT is giving to demonstrate the algorithm and its potential applications. The result indicates that the algorithm can generate the feasible train timetable and solve the conflicts effectively.
\end{abstract}

Keywords: ant colony 0ptimization, train timetabling problem, mass rapid transit.

\section{Introduction}

The train timetabling problem(TTP) aims at determining a periodic timetable for a set of trains that does not violate track capacities and some operation constraints[1].The main methods used in the previous research of TTP can be divided into mathematical model[2,3], simulation method[4] and heuristic algorithm[1,5,6,7]. The mathematical programming method can find the optimal solution, but it will be hard to get solution due to the scale of problem. The simulation method is aimed at examining the factor needed in timetabling by simulating the operation in train system so that interrelationship between different systems can be informed, it can effectively deal with the arrival and departure time of trains, the speeding up and slowing down of trains, but due to the complexity of train system, its operation can be simulated only when all the interface characteristic of systems are grasped. Heuristic algorithm is a designed with regular thinking. Normally, a initial solution is found out, then the objective improvement is made according to this solution. Despite that, with this method, the optimal solution may not be found, an acceptable approximate solution can be obtained with acceptable time. In view of the timeliness in timetabling, this method can be considered relatively efficient. Since the TTP is NP-hard, it's usually impossible to solve large-scale problem within acceptable time. Therefore, this research propose a heuristic algorithm to generate a train timetable of rapid transit system.
The TTP of MRT is how to decide the combination of trains for arrival and departure as well as the conflict resolving on the precondition of the least change in headway. This research is aimed at developing a heuristic algorithm by making use of ant colony optimization (ACO) extensively applied in solving combination optimization in recent years.

The conception of ACO originated from ant system which was put forward by Dorigo in 1991. Dorigo et al.[8] mentioned that the ants in nature would leave pheromone on their passing path. With the odor of pheromone, those ants who are almost blind find the way to the place where there's food and its returning path. When there are over two routes for choice, ants are apt to choose the one with more pheromone and will leave their own pheromone in this path, so most ants will choose the same path after a period of time. This research made the calculation with the above characteristic.

\section{Time-space Diagram}

Previously, ACO was not applied in TTP. In order to improve the application of ACO for TTP, we first transfer the MRT network to time-space diagram network with the conception of time. To establish a time-space diagram should define the node first be combined with the conception of time. Some important nodes include:

(1) depot: place where trains are preserved in operation route, trains leave from depot to main line in peak period, the trains return to depot from main line in off-peak period.

(2) terminal station: located in the two extreme points of main line. The time of the train from its arrival to the departure of the terminal station is defined terminal time. Conflict can be resolved by the adjustment of terminal time.

(3) the joint point of main line and the branch line of depot: in this place, trains operating in main line will conflict with the train which leave or return to the depot in the branch line.

The time-space diagram is showed in Figure 1. The ordinate axis represents the related stations. The abscissa axis represents the node formed with fixed time interval; the link represents the possible route for train including the route between depot and terminal station, stopping in the terminal station and 
that between two terminal stations.

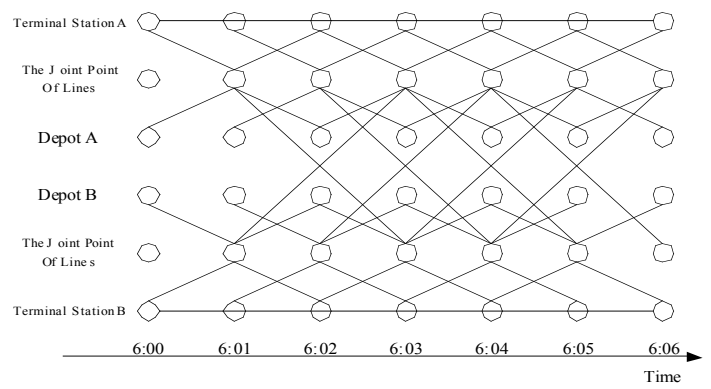

Fig. 1: Time-space diagram

\section{Heuristics based on ACO}

The train timetabling of rapid transit is aimed at making a timetable with no conflict and steady headway [3,7]. With the transition probability of $\mathrm{ACO}$, the algorithm is to choose the train departure time from the terminal station so as to avoid the departure time which will possibly cause conflict and to gradually converge the solution to optimization by pheromone area updating rule and global updating rule.

The core of the algorithm in the research is on the basis of the algorithm in solving TSP put forward by Dorigo et al.[8,9]. There are two important mechanisms in the algorithm: transition probability and evaporation mechanism. The detail is explained as follows:

(1) Transition probability: this mechanism decides the probability in the transition of arrival train to the departure time. In equation (1), $p_{i j}^{s}(t)$ represents the probability of the sth ant choosing departure time $\mathrm{j}$ as next train at the arrival time $\mathrm{i}$ in the $\mathrm{t}^{\text {th }}$ iteration. $J_{s}(\mathrm{i})$ represents the set of departure time which can be chosen in transition for the $s^{\text {th }}$ ant at the arrival time $\mathrm{i}$; $\tau_{j}(t)$ represents the pheromone concentration of the departure time $\mathrm{j}$ in the $\mathrm{t}^{\text {th }}$ iteration; $\eta_{j}$ represents the reciprocal of the numbers of conflicts coursed by departure in the departure time $\mathrm{j} ; \alpha$ and $\beta$ respectively represent the important relative factor of pheromone concentration and conflict frequency

$$
p_{i j}^{s}(t)=\left\{\begin{array}{cc}
\frac{\left[\tau_{j}(t)\right]^{j}\left[\eta_{j}\right]}{\sum_{u \in J_{s}(i)}\left[\tau_{u}(t)\right]^{k}\left[\eta_{u}\right]^{\beta}} & \text { if } j \in J_{s}(i) \\
0 & \text { otherwise }
\end{array}\right.
$$

(2) Evaporation mechanism: there are two rules of updating for the research: local updating rule and global updating rule. The local updating rule is showed in equation (2):

$\tau_{j}(t+1)=\tau_{j}(t)+\rho \tau_{j}^{0}$

Pheromone will be updated when one set of timetable is constructed. In it, $\tau_{j}(t)$ represents the pheromone reside in the $\mathrm{t}^{\text {th }}$ iteration at the departure time $j$; $\rho$ represents the evaporate speed of pheromone $(0 \quad \rho \quad 1) ; \quad \tau_{j}^{0}$ represents the initial value of pheromone at the point $j$; the closer to the scheduled departure time, the higher the initial value, the farther away from the scheduled departure time, the lower the starting value $\left(0<\tau_{j}^{0} \quad 1\right)$. The part of global updating rule is showed in equation (3) to equation (5).

$$
\begin{aligned}
& \tau_{j}(t+1)=(1-\rho) \tau_{j}(t)+\rho \Delta \tau_{j} \\
& \Delta \tau_{j}= \begin{cases}\frac{Q}{L^{+}} & \text {if } j \in T^{+} \\
0 & \text { otherwise }\end{cases} \\
& L=C f \times M+\sum\left[\left(H w_{i}-H w_{i-1}\right)-\left(H w_{i-1}-H w_{i-2}\right)\right]
\end{aligned}
$$

In it, $\Delta \tau_{j}$ is the pheromone concentration of the currently optimized transition combination, as showed in equation (4). In the equation, $Q$ is a constant, representing the total pheromone amount of one ant, $\mathrm{T}+$ is the currently best transition combination, $\mathrm{L}+$ is the total cost; equation (5) represents the total cost of each timetable, this mode is aimed at minimizing the total cost, in the equation, $C f$ is the conflicts, $M$ represents a big $\mathrm{M}$ to minimize conflicts; $\sum\left[\left(H w_{i}-H w_{i-1}\right)-\left(H w_{i-1}-H w_{i-2}\right)\right]$ represents the sum of headway difference, $H w_{i}$ represents the train departure time from the terminal station. The smaller the sum of headway difference is, the better the solution is.

The procedures of the algorithm are showed in Figure2. The detail is explained as follows:

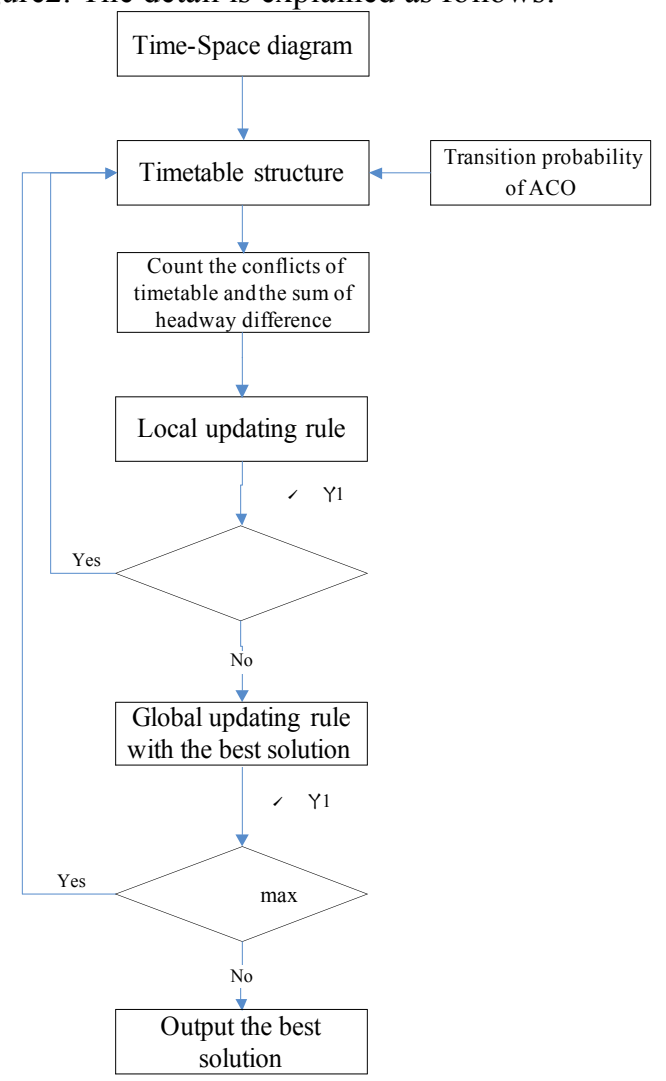

Fig. 2: the flow chart of algorithm

Step 1: set the parameter of train timetabling: the 
related parameter is as follows:

(1) the departure time of the first train and the last train every day.

(2) the roundtrip time between the terminal stations, the time between the depot and the terminal station, the time from the joint point to depot and terminal station.

(3) dwell time of peak period and off-peak period: due to the difference headway in peak and off-peak period, the dwell time of peak and off-peak period should be calculation.

(4) the official headway in peak an off-peak period: the headway should comply with the headway limitation in peak and off-peak period regulated in train operation scheme.

(5) depot capacity and the amount of trains available: besides the consideration in the amount of trains available and depot capacity, consideration should also be taken into that each day, in every depot, the numbers of runs in the morning should be retrieved in the evening.

Step 2: the timetabling of train arrival and departure in each terminal station: set the departure time of each terminal station and estimate the time of arriving at another terminal station according to the headway in each period. There will be an initial timetable of train arrival and departure in each terminal station, which still does not take the train transition conflict resolving into consideration.

Step 3: the parameter setting of ACO algorithm:

(1) The starting value of pheromone $\left(\tau_{j}^{0}\right)$.

(2) Evaporation value of pheromone $(\rho)$.

(3) Numbers of on group of ants (B).

(4) $\alpha$ value and $\beta$ value

(5) the generation $(G)$ : decide the number of t.he group of ants, that is, the working time of ACO.

Step 4: construct transition combination of trains

(1) Progressively scan the time of terminal station in order to distinguish whether it needs trains for departure.

(2) If there needs trains for departure, it should be first examined whether there are trains available for transition. If there is, it can be directly connected and the departure time can be decided with the transition probability of ACO; if there isn't, then train can be departed in depot and be connected in terminal station. In this way, the departure time from terminal station can also be decided with transition probability. As showed in Figure 3, the number in the circle represents the pheromone concentration, when train arrives at the terminal station, choose the time for departure from terminal station with transition probability.

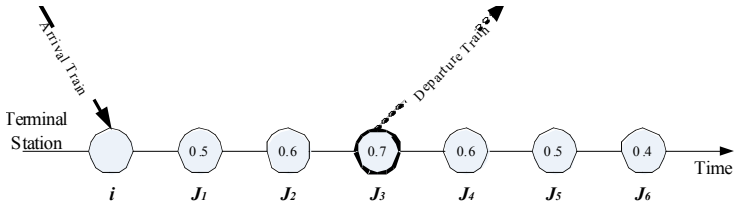

Fig. 3: the illustration of train transition probability

(3) If there is more than one train available for transition, the one which arrives at the terminal station earlier should be retrieved into the depot so as to avoid occupying the platform of terminal station.

(4) By orderly completing the train transition combination in peak and off-peak period, and one group of timetable can be obtained which is the solution from one ant.

(5) Calculate the performance of this group of timetable.

Step 5: local updating of pheromone. When each ant finishes connecting one train combination, the local updating of pheromone in all the paths is made, see equation (2) for the way of updating. Repeat step four until the whole group of ants finish their work.

Step 6: global updating of pheromone. When all the ant in one group finish all their journey, make pheromone updating for the currently optimized transition combination in way of global updating rule. The currently optimized transition combination is not necessarily available in this group of ants, maybe it's what has been searched in the previous group of ants. As to the way of updating should be see Eq.(3), Eq.(4), Eq.(5).

Step 7: update the currently best combination and make a new generation by processing the time.

Step 8: distinguish whether the stop condition has been met, that is, whether all the groups of ants have finished their search journey, and then output the best solution.

\section{Empirical investigation}

\subsection{Real world example}

In the research, ACO algorithm was constructed. In order to validate its accuracy and efficiency of the application in solving the train timetabling problem, we made an empirical investigation of Taipei MRT TamShui and Hsintien, including 30 stations (2 terminal stations), 2 depots. The heuristic algorithm was programmed by Microsoft Visual $\mathrm{C}++$.NET with the PC of AMD 3.0G, 1GB RAM.

The previous task of the research referred such related consideration of timetabling as the station, the arrangement of the railway, the location of depot and operation time of rapid transit system and the line structural diagram was made (as showed in Fig.4). The network time-space diagram was made with the structural diagram for each time interval. In the research, the time interval was set as 1 second. The node was respectively set in related stations 
(terminal station, depot) according to the time interval. The link was set between all related stations according to the potential operation situation. With the scope of test, there are all together 2 depots, 2 terminal stations with over 216000 nodes and 273000 links.

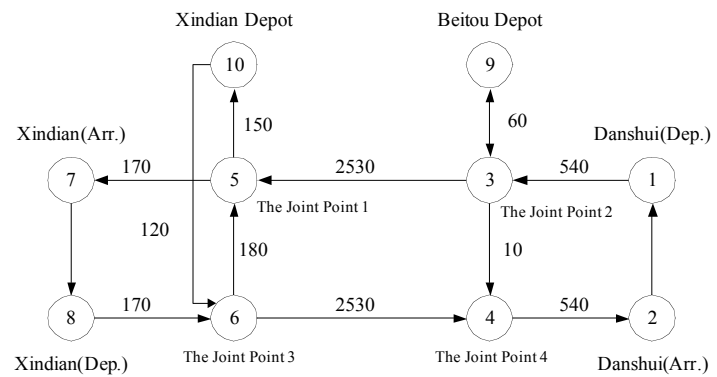

Fig. 4:Taipei MRT Danshui Line ---Xindian Line structural diagram

\subsection{Computational results}

In order to evaluate the performance of ACO's application in TTP of MRT, the research took that of $\mathrm{Su}$ et al.[3] for reference and adopted three evaluation indicators, that is, the proper proportion of headway, the range of headway and the its standard deviation in peak and off-peak period. The result is showed in Table 1.

Table 1: the comparison chart of evaluation indicators in timetable

\begin{tabular}{l|c|c}
\hline \multicolumn{1}{c|}{ indicator } & this research & $\begin{array}{c}\text { Expert of } \\
\text { MRT }\end{array}$ \\
\hline $\begin{array}{l}\text { Proper proportion } \\
\text { of headway }\end{array}$ & $98.52 \%$ & $70.56 \%$ \\
\hline Range of headway & 220 seconds & 442 seconds \\
\hline $\begin{array}{l}\text { Standard deviation } \\
\text { of headway in peak } \\
\text { period }\end{array}$ & 26.15 seconds & 49.03 seconds \\
$\begin{array}{l}\text { Standard deviation } \\
\text { of headway in } \\
\text { off-peak period }\end{array}$ & 14.92 seconds & 63.53 seconds \\
\hline
\end{tabular}

In the process of solving, it was found out that ACO algorithm could rapidly converge into the approximate optimized solution with the average solving time with about $130 \mathrm{~min}$. The analysis of computational result is as follows:

The proper proportion of headway was over $98 \%$. The average range of headway was 220 seconds, showing the rather small headway variation of the timetable by ACO. As to the standard deviation of headway, the computation result was almost within 30 seconds. In general, the above analysis showed that a fairish result could be obtained with the algorithm in the research whether in view of the proper proportion of headway, the headway range and its standard deviation in each period.

\section{Conclusions}

ACO boasts the characteristic of rapidly converging into the approximate solution and keeping away from the local optimized solution. Therefore, this research made use of ACO's advantage in rapid solving combination optimization problem to transfer the network of MRT into time-space diagram and the ACO algorithm was proposed which is applicable in TTP. This algorithm makes selection in train transition combination by transition mechanism and improves the selection probability of the train combination with better target value by means of pheromone updating mechanism. Through the empirical investigation of Taipei MRT Danshui Line ---Xindian Line, it was found out that within 3 hours, the ACO algorithm was able to create a timetable with the proper proportion of headway reaching 99\% and the headway range within 200 seconds, showing that this method could be effectively applied in TTP of MRT.

\section{Reference}

[1] Caprara, A., Fischetti, M. and Toth, P.(2001),"Modeling and solving the train timetabling problem", Operations Research, Vol.50, No. 5, pp.851-861.

[2] Higgins, A., Kozan, E. and Ferreira, L. (1996), "Optimal Scheduling of Trains on A Single Line Track", Transportation Res.-B, Vol.30, No. 2, pp.147-161.

[3] Jau-Ming Su, Chia-Lung Huang, Yu-Chen Huang (2002), "A Two-Stage Method for the Construction of Multi-Depot MRT Timetables", Journal of the Chinese Institute of Transportation, pp. 83-102.

[4] Yu Cheng(1996), "Optimal train traffic rescheduling simulation by knowledge-based system combined with critical path method," Simulation Practice and Theory 4, pp. 399-413.

[5] Cai, X. and Goh, C.J.(1994), “A Fast Heuristic for the Train Scheduling Problem", Computers and Operations Research, V.21, pp. 499-510

[6] Mees A. I. (1991), "Railway Scheduling by Network Optimization", Mathl Comput. Modeling, Vol.15, No.1, pp.33-41.

[7] Ya-Hui Yu (2000), "The Study of Mass Rapid Transit Train Scheduling Problem-A Case Study of Taipei MRT TamShui and Hsintien line", Thesis Submitted to Institute of Transportation Engineering \& Management National Chiao Tung University.

[8] Dorigo, M., Maniezzo, V. and Colorni, A. (1996),"Ant system: optimization by a colony of cooperating agents.",IEEE Transactions on Systems, Man, and Cybernetics-part B: Cybernetics, Vol.26, No.1, pp.29-41.

[9] Dorigo M. and L.M. Gambardella (1997),"Ant Colonies for the Traveling Salesman Problem.", BioSystems, Vol.43, pp.73-81. 